\title{
Myotonic dystrophy (DM) protein kinase levels in congenital and adult DM patients
}

\author{
Monica A Narang ${ }^{1}$, James D Waring ${ }^{1}$, Luc A Sabourin ${ }^{2}$ and Robert G Korneluk ${ }^{1,3}$ \\ ${ }^{1}$ Soulange Gauthier Karsh M olecular Genetics Laboratory, Children's Hospital of Eastern Ontario, Ottawa; \\ ${ }^{2}$ Neuroscience Research Institute and Cellular and M olecular M edicine, University of Ottawa, Ottawa; ${ }^{3}$ Department \\ of Biochemistry, Microbiology and Immunology, University of Ottawa, Ontario, Canada
}

Myotonic dystrophy is caused by a (CTG) $)_{n}$ trinucleotide repeat expansion located in the $3^{\prime}$ untranslated region of the myotonic dystrophy protein kinase gene (DMPK). To date, the disease mechanism has proven elusive. The mutation would not be expected to affect kinase function and yet the disease is inherited in a dominant fashion. Mutant DMPK transcripts have been demonstrated to be retained in affected cell nuclei which could reduce DMPK protein levels and cause disease by haploinsufficiency. An alternate hypothesis is that the expansion confers a toxic gain of function on the transcript. In previous studies, various 52-55 kDa proteins have been detected using antisera targeted against DMPK and a decline of two of these candidates in disease tissues was reported. Current information now suggests that these proteins are not products of the myotonic dystrophy gene. We have characterised an antiserum which has been confirmed to recognise authentic 71 and $80 \mathrm{kDa}$ isoforms of DMPK. Determination of the kinase levels in disease tissues with controls for patient age and tissue integrity demonstrates a modest overexpression in adult patients. In tissues from severely affected congenital patients only a slight decline is seen. This data argues against DM PK haploinsufficiency as a disease mechanism. European Journal of Human Genetics (2000) 8, 507-512.

Keywords: myotonic dystrophy; DMPK; trinucleotide repeat; haploinsufficiency; gain of function

\section{Introduction}

Myotonic dystrophy (DM) is an autosomal dominant neuromuscular disorder characterised by extreme pleiotropism and variability in disease expression. ${ }^{1} \mathrm{DM}$ is caused by the expansion of a (CTG) $)_{n}$ trinucleotide repeat located in the $3^{\prime}$ untranslated region (UTR) of a protein kinase gene (DM PK). ${ }^{2-4}$ Increasing mutant CTG repeat lengths are correlated with increasing disease severity and an earlier age of onset. ${ }^{5}$ The typical form has an onset in early adult life and is characterised by myotonia, skeletal muscle weakness and wasting, cardiac conduction defects and cataracts. A congenital form is frequently fatal and presents with severe hypotonia, respiratory distress and retardation.

\footnotetext{
Correspondence: RG Korneluk, Department of Biochemistry, Microbiology and Immunology, University of Ottawa, Ontario, Canada, K1H 8M5 Tel: +1613738 3281; Fax: + 16137384833 ; E-mail: Bob@mgcheo.med.uottawa.ca Received 22 October 1999; revised 10 February 2000; accepted 1 March 2000
}

Three mechanisms consistent with the dominant mode of inheritance have been proposed. First, expansion might interfere with transcription or post-transcriptional processing of mutant transcripts, resulting in DMPK levels in the range of $50-100 \%$ of normal and causing disease by haploinsufficiency. ${ }^{6-9}$ Second, expansion may confer some novel, toxic gain of function on mutant transcripts. ${ }^{10}$ Third, expansion of the CTG repeat has been associated with the loss of a $3^{\prime}$ DNAse I-hypersensitive site. ${ }^{11}$ Repeat expansion might therefore interfere with the expression of genes adjacent to DMPK (the SIX $5^{12}$ gene lies immediately downstream and 59/DMW $D^{13}$ immediately upstream) by a change in chromatin structure and/or directly occlude enhancer/ promoter elements for SIX 5, contributing to disease according to the tissue-specific expression of these genes. ${ }^{14-16}$

Haploinsufficiency has been controversial because it seems inconsistent with the wide range of disease severity ${ }^{17}$ and because DMPK nullizygous mice do not display a DM-like pathology in skeletal muscle, ${ }^{18,19}$ although cardiac conduction defects similar to those seen in DM were recently 
reported in one strain. ${ }^{20}$ Also, we and others found no decrease in total DMPK transcript levels, in contrast to previous reports. ${ }^{21,22}$ Much of this variability in total transcript measurements is probably explained by the recent demonstration that mutant message accumulates in extraction-resistant foci in the nucleus. ${ }^{23,24}$ In any case, it is now clear that this nuclear retention results in a depletion of mutant DMPK message in the cytoplasm, ${ }^{10,22}$ which could result in a decline of DMPK. Alternatively, mutant transcript foci are also consistent with a transcript gain of function mechanism. Accumulated DMPK transcript could in some way interfere with the processing of other transcripts in trans ${ }^{25}$ and/or sequester CUG repeat-binding proteins with regulatory roles in transcript processing. ${ }^{26}$

Originally, the DMPK protein product (predicted size $69 \mathrm{kDa}$ ) was identified as a protein(s) in the size range of $52-55 \mathrm{kDa}$, as well as a variety of other species. ${ }^{27}$ Reduced expression in DM tissues was reported in two of these studies. ${ }^{6,28}$ However, recent observations have demonstrated that some, if not all, of these candidates are cross-reactive species. ${ }^{18,27}$ We and others have characterised an antiserum which detects two 71 and $80 \mathrm{kDa}$ isoforms which had identical mobility with products of expressed cDNAs, were detected in the appropriate tissue-specific manner, and were dramatically overexpressed in DMPK transgenic mice and absent in DMPK nullizygous mice. ${ }^{18,29-31}$ Similarly, a doublet of 72 and $80 \mathrm{kDa}$ was determined to be authentic DMPK by epitope mapping using a panel of monoclonal antibodies. ${ }^{27}$ Together, these studies are conclusive that our antiserum recognises DMPK products.

Cytoplasmic DMPK transcript levels in a selection of patient cell lines with expansions of up to $4 \mathrm{~Kb}$ were determined to be 56 to $85 \%$ of normal (no reduction in the normal allele was seen). ${ }^{24}$ The critical unanswered question is then whether this is sufficient to affect the steady state DMPK levels. DMPK levels were therefore quantified in skeletal muscle extracts from congenital and adult DM patients and the results were expressed relative to age-matched normal controls. We found that after normalization to a marker for typel musclefibres (which undergo selective atrophy in DM), DMPK is expressed in adult patients at 1.2 to 1.6 times the normal level. In congenital patients, DMPK is expressed at levels only slightly below normal. These results strongly argue against haploinsufficiency as a mechanism of pathogenesis in DM.

\section{Materials and methods Tissues}

All tissues were obtained by courtesy of J Puymirat, Department of Human Genetics, CHU Laval Research Center, SteFoy, Quebec. Skeletal muscle (forearm) was retrieved postmortem from four congenital DM patients at days2, 3, 5 and 6 post-natal from complications due to respiratory distress (designated CDM1 to CDM4, respectively). Expansion sizes were estimated from genomic Southern blots of patient tissue at 520,800,910 and 590 CTG repeats, respectively (data not shown). As controls, post-mortem skeletal muscle was retrieved from four newborns at days 2, 3, 6 and 8 postnatal (designated $n 1$ to $n 4$, respectively). Cause of death was listed as diaphragmatic hernia or congenital heart disease.

Adult skeletal muscle samples (forearm) were obtained during routine diagnostic biopsy. Four patients with DM were examined: a 37 year old male (DM 1), a 53 year old male (DM2), a 56 year old female (DM3) and a 58 year old male (DM4). All DM patients suffered from progressive muscle weakness and diffuse myotonia. Expansions in the E1 range ${ }^{5}$ were confirmed for all patients by Southern blot analysis (data not shown). For controls, four non-DM males aged 32, 48,52 and 60 years at the time of biopsy were used (designated N1 to N4, respectively). All muscle samples were quickly frozen in isopentane, cooled in liquid nitrogen, and stored at $-80^{\circ} \mathrm{C}$ until assayed.

\section{Primary myoblast cultures}

Primary myoblast cultures were established from post-mortem muscle samples from a DM foetus (at 28 weeks) with an expansion estimated at 1000 repeats and from two 15-weekold congenital patients both with expansions estimated at between 3000 to 5000 repeats. As a control, myoblasts were established from a needle biopsy on a 13-month-old, unaffected individual. Muscle was minced into small pieces in cold PBS, then incubated in trypsinisation solution $(0.25 \%$ trypsin, $53 \mathrm{mM}$ EDTA (Gibco/BRL, Rockville, MD, USA) supplemented with $10 \mathrm{~mm}$ glucose) at $37^{\circ} \mathrm{C}$ with shaking for $1 \mathrm{~h}$. Debris was allowed to settle, and the cells in the supernatant were centrifuged at $400 \times \mathrm{g}$ and subjected to a second trypsinisation. The supernatant containing satellite myoblasts were plated and were maintained in F-12 medium containing $20 \%$ foetal bovine serum and $5 \mu \mathrm{g} / \mathrm{ml}$ epidermal growth factor (Sigma, St Louis, MO, USA). The purity of the cultures was determined by immunofluorescence staining for $\mathrm{N}-\mathrm{CAM}$ and desmin and determined to be greater than $98 \%$.

\section{Protein extracts and immunoblot analysis}

Samples were prepared and analysed as described. ${ }^{30} 30 \mu \mathrm{g}$ of total protein from muscle or $20 \mu \mathrm{g}$ from primary myoblasts were electrophoresed and transferred to nitrocellulose. Blotted membranes were incubated for $1 \mathrm{~h}$ with one of the following primary antibodies: anti-DMPK polyclonal ${ }^{30}$ antibody at 1:2500, dilution, anti- $\alpha$-sarcomeric actin monoclonal antibody (5C5; Sigma) at 1:5000, anti-sarcomeric myosin heavy chain ( $\mathrm{MHC}$ ) monoclonal hybridoma supernatant (clone MF20; courtesy of Dr P Merrifield, University of Western Ontario) at 1:10, or anti-typel MHC monoclonal hybridoma supernatant (clone4A9; courtesy of Dr P Merrifield, University of Western Ontario) at 1:10. Immunoreactive bands were visualised using horseradish peroxidasecoupled secondary antibody (anti-mouse or anti-rabbit IgG; 
Amersham) at 1:3000 and enhanced chemiluminscent detection (ECL; Amersham, Uppsala, Sweden). Signals were detected using Hyperfilm ECL (Amersham) in a series of exposures of 1-5 min. All audioradiographs were analysed by laser densitometry (Bio-Rad GS-670 imaging densitometer, Hercules, CA, USA), and a standard exposure time found to be in the linear detection range was selected for each antibody. The values for the 71 and $80 \mathrm{kDa}$ isoforms were added together to give the total DMPK value.

\section{Results}

As previously reported, two protein species were detected in human skeletal muscle extracts using a polyclonal antiDM PK serum (Figures 1 and 2), the larger of which ( $80 \mathrm{kDa})$ is predominant. ${ }^{29,30}$ We measured the apparent DMPK levels in adult patient muscle, expressing the results relative to a normal control matched to within 5 years of age. A significant reduction was seen in all cases (Figure 1A and Table1; Student's 2-tailed t-test, $P<0.005)$. We also measured $\alpha$-sarcomeric actin, total (sarcomeric) M HC and typel-MHC levels to control for the degeneration of diseased muscle. A small decrease, which was not statistically significant, was seen in $\alpha$-actin levels, while there was a significant reduction of total

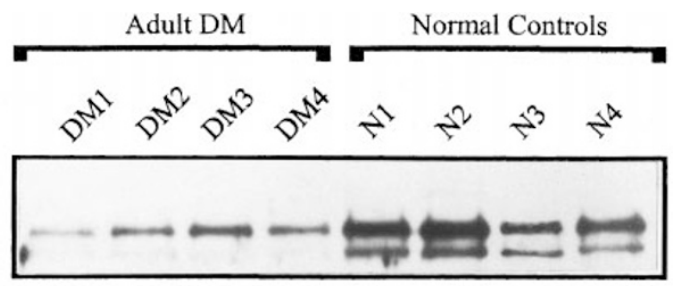

(B) Actin

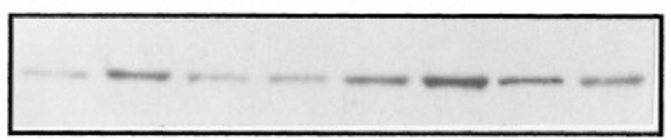

(C) Total Myosin

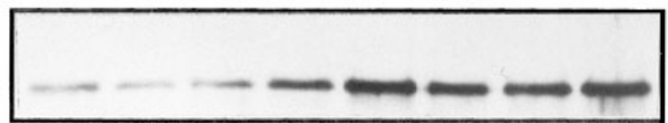

(D) Type I

$\mathrm{MHC}$

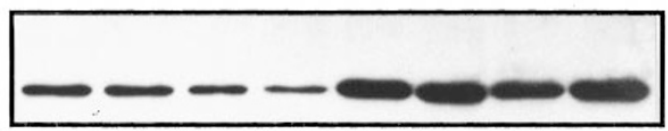

Figure 1 Immunoblot analysis of adult DM skeletal (forearm) muscle from patients DM1 to DM4 and normal age-matched controls N1 to N4 (see Materials and methods) using antibodies against A: DMPK, B: $\alpha$-sarcomeric actin, C: total (sarcomeric) MHC and D: typel-MHC. All patients had confirmed repeat expansions (by muscle biopsy) and presented with progressive muscle weakness and diffuse myotonia. Two DMPK species (71 and $80 \mathrm{kDa}$ ) are seen in A, with the larger predominating. The smaller isoform is very faint for the DM samples (DM1-DM4). A significant deficit of DMPK protein, total MHC and typel-MHC was found in adult DM patients compared to normal controls from values obtained from three replicate blots of the same samples (Table 1; Student's 2-tailed t-test, P <0.005).

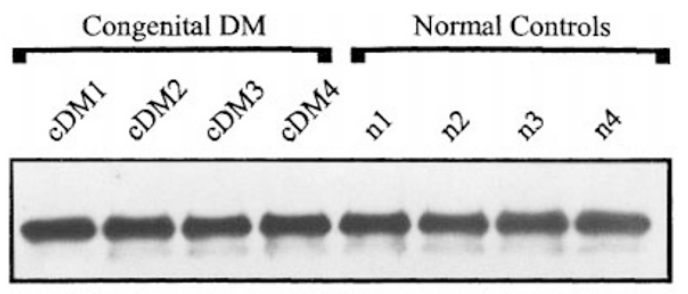

(B) Actin

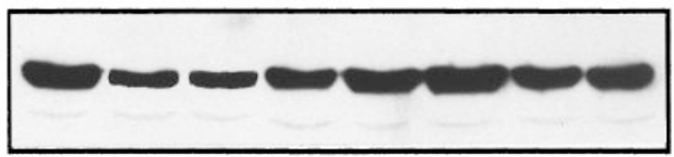

(C) Total Myosin

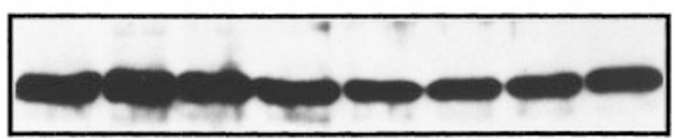

(D) Type I MHC

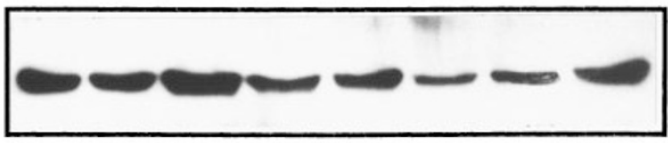

E

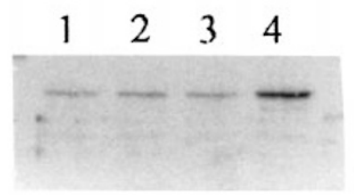

Figure 2 A-D: Immunoblot analysis of congenital DM skeletal (forearm) muscle from patients CDM 1 to CDM 4 and normal age-matched controls $\mathrm{n} 1$ to $\mathrm{n} 4$ as for Figure 1 . All patients died of complications from respiratory distress and had expansions in the congenital range on autopsy. No difference was observed in DMPK expression levels between congenital and normal samples. Total MHC levels appeared to be slightly elevated. E: DMPK immunoblot analysis of primary myoblast cultures established from a 13-month-old unaffected individual (lane 1), a DM fetus at 28 weeks (lane2) and two 15-week-old CDM patients (lanes 3 and 4). The foetal DM and one CDM culture showed no decline in DMPK compared with a normal culture (lanes 1-3), while the other CDM culture had elevated DMPK levels. Only the $80 \mathrm{kDa}$ species is detected in $\mathbf{E}$, while only a small amount of the $71 \mathrm{kDa}$ species is seen in A-D.

MHC and typel-MHC levels (Figure $1 B$ to $D$ and Table1; Student's 2-tailed t-test, $\mathrm{P}<0.005)$. This is consistent with the characteristic skeletal muscle wasting associated with DM. ${ }^{1}$ Compared with levels prior to normalisation, an approximately two-fold increase in relative DMPK levels was seen after normalisation to $\alpha$-actin. Strikingly, upon normalisation to total MHC and/or typel-MHC, DMPK levels showed a small but consistent increase over normal control levels (Table1). The values after normalisation to typel-MHC were higher than for total MHC for all four patients, consistent with selective typel fibre atrophy. ${ }^{32}$

In contrast to adult DM samples, no change was observed in DMPK levels in skeletal muscle samples from four congenital DM patients compared with normal age-matched 
Table 1 Ratio of DMPK levels in skeletal muscle samples from DM patients compared with normal controls

\begin{tabular}{|c|c|c|c|c|c|c|c|c|}
\hline \multirow[b]{3}{*}{ Normalised } & \multirow[b]{3}{*}{1} & \multicolumn{6}{|c|}{ DM PK protein levels in DM patients/normal controls } & \multirow[b]{3}{*}{4} \\
\hline & & \multicolumn{3}{|c|}{ Adult DM patients } & \multirow{2}{*}{\multicolumn{3}{|c|}{$1 \quad 2^{\text {Congenital DM patients }}$}} & \\
\hline & & 2 & 3 & 4 & & & & \\
\hline & $0.21 \pm 0.03$ & $0.30 \pm 0.02$ & $0.32 \pm 0.07$ & $0.25 \pm 0.03$ & $1.00 \pm 0.09$ & $1.01 \pm 0.10$ & $1.09 \pm 0.12$ & $1.03 \pm 0.06$ \\
\hline Actin & $0.46 \pm 0.05$ & $0.49 \pm 0.06$ & $0.51 \pm 0.03$ & $0.58 \pm 0.09$ & $0.96 \pm 0.04$ & $0.91 \pm 0.08$ & $0.95 \pm 0.04$ & $0.93 \pm 0.06$ \\
\hline MHC & $1.09 \pm 0.06$ & $1.15 \pm 0.03$ & $1.10 \pm 0.04$ & $0.97 \pm 0.06$ & $0.74 \pm 0.07$ & $0.80 \pm 0.03$ & $0.83 \pm 0.05$ & $0.90 \pm 0.10$ \\
\hline Type I-MHC & $1.21 \pm 0.09$ & $1.36 \pm 0.11$ & $1.47 \pm 0.08$ & $1.60 \pm 0.21$ & $0.82 \pm 0.04$ & $0.79 \pm 0.06$ & $0.91 \pm 0.05$ & $0.96 \pm 0.08$ \\
\hline
\end{tabular}

DMPK protein levels in DM patients and age-matched controls were determined by laser densitometry. Values are the average of three replicate determinations from each muscle extract in arbitrary units. Results are expressed as the mean ratio + - SD of patient levels to the corresponding agematched control. Ratios are also given after the prior normalisation of DMPK levels to sarcomeric actin, total (sarcomeric) myosin heavy chain (MHC) or type I-MHC determined in parallel experiments using the same muscle extracts.

neonate controls (Figure2A, B and Table1), even after normalisation to $\alpha$-actin. When normalised to either total MHC or type I-MHC, DMPK levels were slightly lower than those of normal controls (Figure2C, D and Table1). Therefore, while congenital DM patients have extremely large CTG repeats and are the most severely affected, only a slight decrease in protein is seen.

To examine this in another way, we established primary myoblast cultures from a DM foetus and two congenitally affected patients. These cultures have the advantage of not being subject to variation in muscle tissue composition due to pathological changes. The control sample available was much older (13months) than the two congenital samples (15 weeks); however it has been determined that the composition of cultures obtained from satellite cells is not affected by the age of the donor or the source of muscle, ${ }^{33}$ that is, that human satellite cells are not predetermined. Therefore, a general comparison of DMPK levels between a normal individual and severely affected patients can be made. Rel ative to total protein loaded, the expression level of DMPK in these lines was found to be much lower than in skeletal muscle. Only the larger of the two isoforms was expressed, similar to what was observed for the congenital DM muscle samples (Figures 2A and E). DM PK levels were similar for the control culture (Figure2E, lane1) and cultures from foetal muscle (lane2) and one congenital patient (lane3). Surprisingly, approximately four-fold higher levels were seen in the culture from the second congenital sample (lane4), despite being derived from a biopsy similar to the first congenital sample (lane3). The basis for this is not known.

\section{Discussion}

After normalisation to MHC-I, adult patients had a small but consistent elevation of DMPK. This demonstrates the importance of controls for the effects of disease on tissue, as previously suggested. ${ }^{17}$ In addition, no decrease was seen in DMPK expression in myoblast lines from cDM patient samples, where tissue integrity is not an issue. More importantly, we found only a minor decrease of DMPK in congenital tissues. While immunoblotting is not a fully quantitative technique, any influence of extreme expansion sizes over DMPK levels would undoubtedly be observed in these samples. As there is clearly a loss of mutant transcript from the cytoplasm in patient tissues, ${ }^{10,22,24}$ our data suggests that declines to $\geq 50 \%$ normal are not reflected at the translational level. Similarly, mice hemizygous for $\alpha$-tropomysosin have $50 \%$ of the normal transcript level, as expected, but normal levels of protein. ${ }^{34}$ Although there possibly may be other DMPK isoforms not detected by our antiserum which remain to be analysed, this data strongly argues against DMPK haploinsufficiency as a disease mechanism in muscle.

The basis for the modest overexpression of DMPK relative to $\mathrm{MHC}$ in adult and not in congenital patients is unknown. Adults typically have progressive muscle degeneration and typel fibre atrophy, while congenital patients are hypotonic. The expression of DMPK may therefore be enhanced in response to the degenerative process. DMPK expression is induced upon conversion of cells to myoblasts ${ }^{11,35}$ and is modestly upregulated upon myoblast differentiation. ${ }^{36}$

A similar increase of a $64 \mathrm{kD}$ a species relative to typel-MHC in affected DM muscle using a monoclonal antibody has been reported. ${ }^{37}$ In contrast, our results differ from those in another study using our antibody. ${ }^{29}$ No decrease in total myosin levels was seen in patient muscle samples in the latter, leading the authors to conclude that DMPK levels were reduced. The latter study differed in that total myosin was assessed by protein staining and the muscles sampled (quadriceps and deltoid) are not affected to the degree that distal limb muscles are. Using specific monoclonal antibodies, we observed a similar decline in total sarcomeric and typel-MHC levels for all four patients tested, consistent with the disease pathology.

It has been proposed that the CUG repeat-binding protein CUG-BP/hNab50 is a substrate for DMPK and that reduced DMPK levels alter the localisation of this factor. ${ }^{26}$ CUG-BP may function in developmentally regulated splicing pathways. ${ }^{38}$ Our data suggests that CUG-BP1 function would not be altered secondary to reduced DMPK levels, although it might be still be affected by repeat expansions. In conclusion, our results support continued efforts in the identification of RNA-binding factors which may mediate a toxic gain of function and further investigation of the roles of genes 
adjacent to DMPK. The possible role of DMPK haploinsufficiency specifically in the heart requires further investigation. ${ }^{20}$

\section{Acknowledgements}

We are grateful to Dr Gillian Butler-Browne for helpful discussions. This work was supported by grants to RGK from the Muscular Dystrophy Associations of Canada and the USA, the Medical Research Council of Canada (MRC) and the Canadian Networks Centres of Excellence. MAN was supported by the Arthur Minden Fellowship from the Muscular Dystrophy Association of Canada. RGK is a recipient of an MRC Scientist award.

\section{References}

1 Harper PS: Myotonic dystrophy. 2nd edn. WB Saunders: London, 1989.

2 Brook JD, McCurrach ME, Harley HG et al: Molecular basis of myotonic dystrophy: expansion of a trinucleotide (CTG) repeat at the $3^{\prime}$ end of a transcript encoding a protein kinase family member. Cell 1992; 69: 799-808.

3 Mahadevan M, Tsilfidis C, Sabourin L et al: Myotonic dystrophy mutation: an unstable CTG repeat in the $3^{\prime}$ untranslated region of the gene. Science 1992; 255: 1253-1255.

4 Fu YH, Pizzuti A, Fenwick RG Jr et al: An unstable triplet repeat in a gene related to myotonic muscular dystrophy. Science 1992; 255: $1256-1258$.

5 Tsilfidis C, MacKenzie AE, Mettler G, Barcelo J, Korneluk RG: Correlation between CTG trinucleotide repeat length and frequency of severe congenital myotonic dystrophy. Nat Genet 1992; 1: 192-195.

$6 \mathrm{Fu} \mathrm{YH,} \mathrm{Friedman} \mathrm{DL,} \mathrm{Richards} \mathrm{S} \mathrm{et} \mathrm{al:} \mathrm{Decreased} \mathrm{expression} \mathrm{of}$ myotonin-protein kinase messenger RNA and protein in adult form of myotonic dystrophy. Science 1993; 260: 235-238.

7 Novelli G, Gennarelli M, Zelano G et al: Failure in detecting mRNA transcripts from the mutated allele in myotonic dystrophy muscle. Biochem Mol Biol Int 1993; 29: 291-297.

8 Carango P, Noble JE, Marks HG, Funanage VL: Absence of myotonic dystrophy protein kinase (DMPK) mRNA as a result of a triplet repeat expansion in myotonic dystrophy. Genomics 1993; 18: $340-348$

9 Hofmann-Radvanyi H, Lavedan C, Rabes JP et al: Myotonic dystrophy: absence of CTG enlarged transcript in congenital forms, and low expression of the normal allele. Hum Mol Genet 1993; 2: 1263-1266.

10 Wang J, Pegoraro E, Menegazzo E et al: Myotonic dystrophy: evidence for a possible dominant-negative RNA mutation. Hum Mol Genet 1995; 4: 599-606.

11 Otten AD, Tapscott SJ: Triplet repeat expansion in myotonic dystrophy alters the adjacent chromatin structure. Proc Natl Acad Sci USA 1995; 92: 5465-5469.

12 Boucher CA, King SK, Carey $\mathrm{N}$ et al: A novel homeodomainencoding gene is associated with a large CpG island interrupted by the myotonic dystrophy unstable (CTG)n repeat. Hum Mol Genet 1995; 4: 1919-1925.

13 Jansen B, Bachner D, Coerwinkel M, Wormskamp N, Hameister H, Wieringa B: Structural organization and developmental expression pattern of the mouse WD-repeat gene DMR-N9 immediately upstream of the myotonic dystrophy locus. Hum Mol Genet 1995; 4: 843-852.

14 Klesert TR, Otten AD, Bird TD, Tapscott S): Trinucleotide repeat expansion at the myotonic dystrophy locus reduces expression of DMAHP. Nat Genet 1997; 16: 402-406.

15 Thornton CA, Wymer JP, Simmons Z, McClain C, Moxley RT 3rd: Expansion of the myotonic dystrophy CTG repeat reduces expression of the flanking DMAHP gene. Nat Genet 1997; 16: 407-409.
16 Alwazzan M, Newman E, Hamshere MG, Brook JD: Myotonic dystrophy is associated with a reduced level of RNA from the DMWD allele adjacent to the expanded repeat. Hum Mol Genet 1999; 8: 1491-1497.

17 Roses AD: Muscle biochemistry and a genetic study of myotonic dystrophy. Science 1994; 264: 587-588.

18 Jansen G, Groenen PJ, Bachner D et al: Abnormal myotonic dystrophy protein kinase levels produce only mild myopathy in mice. Nat Genet 1996; 13: 316-324.

19 Reddy S, Smith DB, Rich MM et al: Mice lacking the myotonic dystrophy protein kinase develop a late onset progressive myopathy. Nat Genet 1996; 13: 325-335.

20 Berul $\mathrm{Cl}$, Maguire CT, Aronovitz MJ et al: DM PK dosage alterations result in atrioventricular conduction abnormalities in a mouse myotonic dystrophy model. J Clin Invest 1999; 103: R1-7.

21 Sabourin LA, Mahadevan MS, Narang M, Lee DS, Surh LC, Korneluk RG: Effect of the myotonic dystrophy (DM) mutation on mRNA levels of the DM gene. Nat Genet 1993; 4: 233-238.

22 Krahe R, Ashizawa T, Abbruzzese $C$ et al: Effect of myotonic dystrophy trinucleotide repeat expansion on DMPK transcription and processing. Genomics 1995; 28: 1-14.

23 Taneja KL, McCurrach M, Schalling M, Housman D, Singer RH: Foci of trinucleotide repeat transcripts in nuclei of myotonic dystrophy cells and tissues. J Cell Biol 1995; 128: 995-1002.

24 Davis BN, McCurrach ME, Taneja KL, Singer RH, Housman DE: Expansion of a CUG trinucleotide repeat in the $3^{\prime}$ untranslated region of myotonic dystrophy protein kinase transcripts results in nuclear retention of transcripts. Proc Natl Acad Sci USA 1997; 94: 7388-7393.

25 Morrone A, Pegoraro E, Angelini C, Zammarchi E, Marconi G, Hoffman EP: RNA metabolism in myotonic dystrophy: patient muscle shows decreased insulin receptor RNA and protein consistent with abnormal insulin resistance. J Clin Invest 1997; 99 1691-1698.

26 Roberts R, Timchenko NA, Miller JW et al: Altered phosphorylation and intracellular distribution of a (CUG) $n$ triplet repeat RNAbinding protein in patients with myotonic dystrophy and in myotonin protein kinase knockout mice. Proc Natl Acad Sci USA 1997; 94: 13221-13226.

27 Pham YCN, Man N, Lam LT, Morris GE: Localization of myotonic dystrophy protein kinase in human and rabbit tissues using a new panel of monoclonal antibodies. Hum Mol Genet 1998; 7: 1957-1965.

28 Koga R, Nakao Y, Kurano $Y$ et al: Decreased myotonin-protein kinase in the skel etal and cardiac muscles in myotonic dystrophy. Biochem Biophys Res Comm 1994; 202: 577-585.

29 Maeda M, Taft CS, Bush EW et al: Identification, tissue-specific expression, and subcellular localization of the $80-$ and $71-\mathrm{kDa}$ forms of myotonic dystrophy kinase protein. J Biol Chem 1995; 270: 20246-20249.

30 Whiting EJ, Waring JD, Tamai $K$ et al: Characterization of myotonic dystrophy kinase (DMK) protein in human and rodent muscle and central nervous tissue Hum Mol Genet 1995; 4: 1063-1072.

31 Waring JD, Haq R, Tamai K, Sabourin LA, Ikeda JE, Korneluk RG: Investigation of myotonic dystrophy kinase isoform translocation and membrane association. J Biol Chem 1996; 271 15187-15193.

32 Soussi-Yanicostas N, Chevallay M, Laurent-Winter C, Tome FM, Fardeau M, Butler-Browne GS: Distinct contractile protein profile in congenital myotonic dystrophy and X-linked myotubular myopathy. Neuromuscl Disord 1991; 1: 103-111.

33 Edom F, Mouly V, Barbet JP, Fiszman MY, Butler-Browne GS: Clones of human satellite cells can express in vitro both fast and slow myosin heavy chains. Dev Biol 1994; 164: 219-229.

34 Blanchard EM, lizuka K, Christe $M$ et al: Targeted ablation of the murine al pha-tropomyosin gene. Circ Res 1997; 81: 1005-1010.

35 Skerjanc IS, Slack RS, McBurney MW: Cellular aggregation enhances MyoD-directed skeletal myogenesis in embryonal carcinoma cells. Mol Cell Biol 1994; 14: 8451-8459. 
36 Storbeck CJ, Sabourin LA, Waring JD, Korneluk RG: Definition of regulatory sequence elements in the promoter region and the first intron of the myotonic dystrophy protein kinase gene. J Biol Chem 1998; 273: 9139-9147.

37 Dunne PW, Ma L, Casey DL, Harati Y, Epstein HF: Localization of myotonic dystrophy protein kinase in skeletal muscle and its alteration with disease. Cell Motil Cytoskeleton 1996; 33: 52-63.
38 Philips AV, Timchenko LT, Cooper TA: Disruption of splicing regulated by a CUG-binding protein in myotonic dystrophy. Science 1998; 280: 737-741. 